\title{
Cellular-Level Characterization of Lymph Vessels in Live, Unlabeled Corneas by In Vivo Confocal Microscopy
}

Beatrice Bourghardt Peebo, Per Fagerholm, Catharina Traneus-Röckert and Neil Lagali

\author{
Linköping University Post Print
}

Tweet

N.B.: When citing this work, cite the original article.

Original Publication:

Beatrice Bourghardt Peebo, Per Fagerholm, Catharina Traneus-Röckert and Neil Lagali, Cellular-Level Characterization of Lymph Vessels in Live, Unlabeled Corneas by In Vivo Confocal Microscopy, 2010, Investigative Ophthalmology and Visual Science, (51), 02, 830835.

http://dx.doi.org/10.1167/iovs.09-4407

Copyright: Association for Research in Vision and Ophthalmology (ARVO) http://www.arvo.org/

Postprint available at: Linköping University Electronic Press http://urn.kb.se/resolve?urn=urn:nbn:se:liu:diva-65887 
Cellular-Level Characterization of Lymph Vessels in Live, Unlabeled Corneas by In Vivo Confocal Microscopy

Beatrice Bourghardt Peebo ${ }^{1}$, Per Fagerholm ${ }^{1}$, Catharina Traneus-Röckert ${ }^{2}$, and Neil Lagali ${ }^{1}$

${ }^{1}$ Department of Ophthalmology, Linköping University Hospital, 58185 Linköping, Sweden

${ }^{2}$ Department of Pathology, Linköping University Hospital, 58185 Linköping, Sweden

Supported by grants from the the Carl and Axel Molins Memorial Foundation (BBP), and a Marie Curie International Research Fellowship (NL).

Disclosure: B. Bourghardt Peebo, None; P. Fagerholm, None; C. Traneus-Röckert, None; N. Lagali, None.

Word count: 2859

Corresponding author and reprint request:

Neil Lagali

Department of Ophthalmology

Linköping University Hospital

58185 Linköping, Sweden

Tel +4613224658

Fax +46 13223065

Email:nlagali@ohri.ca 


\section{ABSTRACT}

Purpose. To determine whether in-vivo confocal microscopy (IVCM) of the cornea can be used for the label-free detection and monitoring of lymph vessels in live corneas.

Methods. Parallel corneal hem- and lymphangiogenesis was induced by the placement of a single suture in one cornea of male Wistar rats. Fourteen days after suture placement and under general anaesthesia, laser-scanning IVCM was performed in the vascularized region. Corneas were subsequently excised for flat-mount double immunofluorescence with a panendothelial marker (PECAM-1/CD31) and a lymphatic endothelial specific marker (LYVE1). Using the suture area and prominent blood vessels as points of reference, the identical microscopic region was located in both fluorescent and archived in-vivo images. Additionally, vessel diameter, lumen contrast, and cell diameter and velocity within vessels were quantified from in-vivo images.

Results. Comparison of identical corneal regions in fluorescence and in-vivo revealed prominent $\mathrm{CD} 31^{+} / \mathrm{LYVE}-1^{+++}$lymph vessels that were visible in-vivo. In-vivo, corneal lymph vessels were located in the vascularized area in the same focal plane as blood vessels, but had a darker lumen $(\mathrm{P}<0.001)$ sparsely populated by highly reflective cells with diameter similar to leukocytes in blood vessels $(\mathrm{P}=0.61)$. Cell velocity in lymph vessels was significantly reduced relative to blood particle velocity $(\mathrm{P}<0.001)$. Morphologic characteristics enabled subsequent identification of corneal lymphatics in live, vascularized rat corneas prior to immunofluorescent labelling.

Conclusions. IVCM enabled the non-destructive, label-free, in-vivo detection of corneal lymphatics. IVCM provides the possibility to observe lymphatic activity in the same live corneas longitudinally, and as a clinical instrument, the additional possibility to monitor corneal lymphatics in live human subjects. 
Key words: cornea, lymph vessel, lymphangiogenesis, neovascularization, in-vivo confocal microscopy

Under normal circumstances, the angiostatic nature of the cornea contributes to the maintenance of its immune privilige. Secondary to disease, trauma, or infection, however, this angiostasis can be compromised and result in corneal neovascularization characterized by the parallel invasion of blood and lymphatic vessels (hem- and lymphangiogenesis), ${ }^{1,2}$ with subsequent loss of corneal clarity and vision and a poorer prognosis for treatment ${ }^{2,3}$ While immune cells enter the cornea via the afferent blood vasculature, ${ }^{4}$ it is the corneal lymph vessels that provide a direct efferent route for antigen-presenting cells to access regional lymph nodes. ${ }^{5}$ The critical role of lymph vessels is further underscored in cancerinduced lymphangiogenesis where the dissemination of tumor cells through lymph vessels is believed to initiate tumor metastasis. ${ }^{6}$ Despite the importance of lymphangiogenesis, however, relatively little is known about the dynamics of lymph vessel formation, growth, and regression, ${ }^{2,7,8}$ or about cellular activity within lymphatics.

Unlike corneal blood vessels which can be easily visualized and assessed by slit-lamp biomicroscopy, corneal lymph vessels have long eluded in-vivo detection due to the transparency of lymphatic endothelial cells and the lymph fluid. As a result, lymphatic research has advanced using vascular endothelial markers such as LYVE-1, Prox1 and Podoplanin, ${ }^{1,7}$ but with the drawback that destructive, ex-vivo analysis precludes longitudinal observation of the same cornea or the study of cell transport within lymphatics. Moreover, current methods of visualizing lymph vessels limit lymphatic research to animal models ${ }^{2-5,7,8}$ or to a retrospective analysis of excised human corneal grafts in failed transplant cases ${ }^{1,9}$. To date, there has been no report of non-destructive, label-free, in-vivo observation of lymphatics, nor of any technique that can be directly applied to the observation of lymphatics in human subjects. We therefore undertook this study to determine whether in-vivo confocal 
microscopy of the cornea, a non-destructive and label-free clinical technique ${ }^{10}$, could be used to identify lymph vessels in their native state in a live cornea.

\section{$\underline{\text { Methods }}$}

Rat model of suture-induced inflammatory corneal neovascularization

Twelve- to 16-week-old male Wistar rats weighing 200 to 400g (Scanbur AB, Sollentuna, Sweden) were used. All animals were treated following ARVO guidelines for of the Use of Animals in Ophthalmic and Vision Research. With approval from the Linköping regional animal ethics review board, rats were anaesthetized using intraperitoneal injection of dexdomitor and xylazine (20 mg/kg body weight and $75 \mathrm{mg} / \mathrm{kg}$ body weight, respectively). Under an operating microscope, a single 10-0 nylon suture was placed through the corneal stroma approximately 1.5 to $2 \mathrm{~mm}$ from the temporal limbus at the 9 o'clock position in the right eye. Suture placement was chosen to limit neovascularization to a well-defined area and to facilitate access to the vessels by the in-vivo confocal microscope. Two days prior to invivo examination, chloramphenicol ointment (Chloromycetin, Pfizer AB) was applied in the sutured cornea (twice in $24 \mathrm{~h}$ ) to reduce the effect of any presumed secondary infection and thereby minimize the potential of developing corneal haze. Fourteen days after the initial surgery, rats were anesthetized and laser scanning in-vivo confocal microscopy of the corneas was performed.

\section{In-vivo confocal microscopy}

With rats under general anaesthesia and in the prone position, corneas were examined with a laser-scanning in-vivo confocal microscope with corneal imaging module (HRT3-RCM, Heidelberg Engineering, Heidelberg, Germany). The microscope was equipped with a $63 \times / 0.95$ NA water-immersion objective (Zeiss), providing an en face view of a $400 \times 400 \mu \mathrm{m}$ 
corneal area at a selectable corneal depth. The instrument was prepared as for a routine clinical corneal examination following manufacturer instructions. Briefly, a large drop of transparent tear gel (Bausch \& Lomb) was placed on the microscope objective lens and a sterile disposable plastic cap (Tomocap, Heidelberg Engineering) was affixed over the gelcoated lens. A second drop of tear gel was placed on the outer surface of the cap and the gelcoated cap was brought into contact with the cornea in the suture region using the HRT3 manual alignment controls. The focus depth of the HRT3 was initially adjusted to image the corneal epithelial surface, and the lateral and transverse microscope alignment were adjusted to locate the suture in the real-time image display window. Once the suture was located, the focal plane was adjusted axially to locate blood vessels. Digital images were recorded at 5 frames/s, and the probed region was adjusted in lateral, transverse, and axial directions during image capture to locate and follow the path of vessels from the suture area to the limbus. A typical corneal examination consisted of 30 to 50 image sequences with each sequence containing 100 successive digital image frames. Sequences could be analyzed frame-by-frame or in video mode, played at the image acquisition rate.

Double immunofluorescence of corneal hem- and lymphangiogenesis

Following in-vivo microscopy, animals were euthanized by intraperitoneal injection of 100 $\mathrm{mg} / \mathrm{kg}$ pentobarbital sodium (Apoteket AB, Sweden) and the entire cornea with scleral rim was excised and prepared for flat-mounting with three short radial cuts. Corneas were immediately rinsed in PBS; fixed in acetone; rinsed in PBS; blocked in 10 \% normal donkey serum (Jackson ImmunoResearch Laboratories); and incubated with LYVE-1 overnight (goat polyclonal, 1:250; Santa Cruz Biotehcnology). The next day, samples were washed and stained with Cy 3 (donkey anti-goat, 1:100; Jackson); washed and stained with PECAM1/CD31 over the following night (mouse monoclonal, 1:100; Santa Cruz); washed and stained 
with Cy2 (donkey anti-mouse, 1:50; Jackson); and analyzed using a confocal laserscanning fluorescence microscope (Nikon Eclipse E600) equipped with a 40×/1.30 NA oilimmersion objective lens (Nikon). The Cy2 secondary antibody fluoresced green under blue laser (488nm) excitation while Cy3 fluoresced red (543nm excitation). Samples were scanned under dual laser excitation and a digital camera was used to record images.

Quantification of in-vivo vessel morphology and statistical analysis

Individual in-vivo image frames of blood and lymph vessels within two rat corneas were analyzed using image processing software ${ }^{11}$. Images with distinct vessels were selected, containing both blood and lymph vessels at the same distance from the limbus, and where possible, at the same corneal depth. Blood and lymph vessel diameter was measured manually using a line tool by two independent observers, with no significant difference detected between observers for blood $(\mathrm{P}=0.16, \mathrm{n}=38)$ or lymph $(\mathrm{P}=0.24, \mathrm{n}=38)$ vessels. Lymph vessel diameter was measured in each vessel segment of differing diameter while blood vessels had a constant diameter and were measured once per vessel. Lymph and blood particle diameter was measured once per cell and only for cells with distinct boundaries. The contrast ratio of the vessel lumen to the surrounding extracellular matrix (ECM) was determined by the ratio of the 8-bit grayscale pixel value at the center of the vessel lumen to the pixel value of the ECM at a distance of one vessel diameter from the center of the lumen in a direction perpendicular to the direction of flow. Cell velocity in lymph vessels represents the velocity of cells that were in motion when images were viewed in sequence (stationary cells were not included). Cell velocity in blood vessels was determined by identifying individual moving cells in the center of blood vessels, and measuring their displacement in two successive image frames (200ms interval) to limit the influence of motion artifacts. 
Statistical comparison of vessel parameters was performed using an independent t-test where values were normally-distributed, and the nonparametric Mann-Whitney rank sum test otherwise. Normality was determined by the Kolmogorov-Smirnov test. All comparisons were performed using statistical software (SigmaStat, Systat Software Inc.) and a two-tailed value of $\mathrm{P}<0.05$ was considered statistically significant.

\section{$\underline{\text { Results }}$}

In-vivo and ex-vivo correlation of corneal neovascularization

Fourteen days following suture placement in one eye of a rat cornea, aggressive corneal neovascularisation was observed, concentrated in the area between the suture and the temporal limbus (Fig. 1a). With the rat under general anaesthesia, the cornea was examined by laser-scanning in-vivo confocal microscopy with the clinical instrument shown in Fig 1b, which provides an en face view, parallel to the corneal surface. Following in-vivo examination, the rat was euthanized and the entire cornea with scleral rim was removed, keeping the suture in place. The full-thickness cornea was then prepared for flat-mounting to preserve the en face view, and then processed for double immunofluorescence using the panendothelial marker CD31/PECAM-1 for blood vessels and the lymphatic endotheliumspecific marker LYVE-1 for lymph vessels.

The suture area was located with a confocal laser-scanning fluorescence microscope, and the focal depth was adjusted to locate vascular structures. Both blood (CD31 $\left.1^{+++} / \mathrm{LYVE}-1^{-}\right)$and lymph $\left(\mathrm{CD} 31^{+} / \mathrm{LYVE}^{+1^{++}}\right)$vessels were observed in the immediate vicinity of the suture (Fig. 2). Concurrently, archived in-vivo confocal microscope images were manually searched to locate the same microscopic region adjacent to the suture. Characteristic blood vessel branches and bends were used as points of reference. Once the identical region was found, a 
direct comparison of in-vivo and ex-vivo images was possible, revealing the in-vivo morphology of both blood-carrying vessels and lymph vessels containing cells (Fig 2).

Prospective, morphologic detection of corneal lymphatics in-vivo

Since a retrospective, manual search of in-vivo images was data-intensive and did not permit observation of lymphatic activity in real-time, we identified specific morphologic features of lymph vessels from Figure 2 that could aid in their prospective in-vivo detection, prior to exvivo analysis. In-vivo, lymph vessels had no discernible vessel walls, a dark lumen, and contained reflective cells (presumed leukocytes) of roughly uniform size, which were larger than the majority of particles observed in blood vessels.

To determine whether corneal lymphatics could be prospectively detected based solely upon these morphologic features, the rat model of corneal angiogenesis was again employed, followed by in-vivo confocal microscopy after 14 days. During in-vivo examination, several vessels with lumens substantially darker than the surrounding extracellular matrix were observed. These vessels (presumed lymphatics) carried reflective cells of approximately uniform diameter (Fig. 3). The vessels were superficially located, at the same corneal depth as blood vessels approximately 41 to $46 \mu \mathrm{m}$ below the corneal epithelial surface. Video sequences depicting the motion of leukocytes within these vessels were also taken (Supplementary video 1 and 2), and revealed a substantially slower movement of cells compared to the flow within blood vessels. After in-vivo imaging, corneas were excised and prepared for immunofluorescence. Fluorescent images of the suture area were used to locate the suspected lymphatics, which were confirmed as lymph vessels by CD $31^{+} / \mathrm{LYVE}-1^{+++}$ expression (Fig 4). Additionally, in a single rat cornea without neovascularization, we located presumed lymphatics normally present in the temporal bulbar conjunctiva, which were similarly subsequently confirmed as conjunctival lymphatics by immunofluorescence (Fig 5). 
Quantitative in-vivo analysis of corneal blood and lymph vessels

Longitudinal observation of corneal lymphatics in-vivo in the same animal or in humans without the use of contrast agents requires a means to objectively distinguish lymph vessels from blood vessels. We therefore quantified from in-vivo images the morphologic characteristics of blood and lymph vessels in the rat cornea (Table 1). While blood and lymph vessel diameter did not significantly differ, lymph vessels appeared to be constructed of multiple adjoining vessel segments of variable diameter, whereas blood vessels appeared continuous with a constant diameter along their length. Blood vessel branches were abrupt, making well-defined angles relative to the vessel trunk, whereas lymph vessel junctions appeared rounded and more continuous. Additionally, lymphatic vessel walls could not be visualized in-vivo. Lymph vessels had a lumen about half as reflective as the surrounding ECM while blood vessels dense with reflective, flowing particles, had a lumen almost twice as reflective as the ECM; the lumen of blood vessels was thus on average four times as reflective as the lymph vessel lumen in the same corneal region. Leukocytes within lymph vessels were of uniform diameter, appeared highly reflective, and did not appear to differ morphologically from leukocytes in blood vessels. Notably, however, cells with leukocyte morphology were the only cells observed in lymphatics while blood vessels contained both smaller particles (presumed erythrocytes; majority of cells, $2-3 \mu \mathrm{m}$ in diameter) and leukocytes (minority). Leukocyte velocity in lymph vessels was significantly lower than the central particle velocity in blood vessels, by a factor of four on average. While leukocytes in blood vessels appeared to flow along with erythrocytes in the vessel center or 'roll' along the inner blood vessel walls ${ }^{12}$, all leukocytes we observed in corneal lymphatics did not move along vessel walls and no visual evidence of rolling could be detected. Additionally within 
lymphatics, leukocytes sometimes appeared stationary within the duration of observation (tens of seconds), and sometimes changed their direction of motion.

\section{$\underline{\text { Discussion }}$}

Advances in non-invasive, in-vivo microscopy have enabled the repeated imaging of live human ${ }^{10}$ and animal ${ }^{10,13}$ tissue without the use of exogenous contrast agents, but with the drawback that images contain purely morphological information without molecular-level specificity ${ }^{10,14}$. While attempts have been made to correlate in-vivo morphology with ex-vivo staining of histologic sections ${ }^{14,15}$ or immunofluorescence from impression cytology ${ }^{16,17}$, so far an exact correlation has not been possible due to the technical difficulty in imaging the identical tissue region in the same anatomical view and with similar resolution ${ }^{14}$ both in-vivo and ex-vivo.

We found that by providing the same en face, high-resolution confocal microscopic view of the same tissue region both in-vivo and ex-vivo, a direct correlation of tissue morphology with molecular expression was possible, leading to the identification of corneal lymph vessels in-vivo. Moreover, the morphology of lymph vessels was highly specific, and enabled subsequent monitoring of lymphatic activity - including the dynamics of leukocyte movement within lymphatics - prospectively and without the use of contrast agents. This result suggests that by repeatedly probing the same tissue region in-vivo, the unique morphologic features of specific lymph vessels (such as their shape and relative location) can be used to longitudinally monitor the same lymph vessel in-vivo.

In this study, corneal lymphatics in their native state appeared morphologically distinct from adjacent blood vessels. Lymph vessels had a dark lumen in-vivo, which is consistent with the transparency of the lymph fluid. Segmented, bulging lymph vessel walls resulted in a variable vessel diameter in-vivo, which is consistent with lymphatic vascular endothelial morphology observed ex-vivo ${ }^{6,7}$. While leukocyte morphology in blood and lymph vessels was similar, 
leukocyte velocity in lymphatics was on average four times as slow as the blood velocity in the inflamed area. It was additionally observed that lymphatic leukocytes changed their direction of motion while others appeared stationary - behaviour in accordance with known oscillations in the flow of lymph ${ }^{18}$. Our simple method of calculating blood velocity by particle tracking, however, may have resulted in an underestimate of the true blood velocity by at least a factor of five ${ }^{19,20}$, as the cells we observed in the blood vessels were likely the slowest and most amenable to observation ${ }^{19}$.

It is expected that the distinct differences in lymph and blood vessel morphology noted in the rat cornea can be applied to the direct detection of human corneal lymphatics given the broad morphological similarity of the cornea across species ${ }^{13}$, and the ability to use the same clinical in-vivo confocal microscope to examine animal and human corneas ${ }^{10,13}$. Although corneal lymph vessels have yet to be observed in a live human subject, presumed conjunctival human lymph vessels have earlier been reported previously with in-vivo confocal microscopy, but without histological proof ${ }^{21}$. It would be of considerable clinical interest to detect lymph vessels in ocular inflammatory conditions or in corneal transplant cases, where lymph vessels play a critical role in the acceptance or rejection of allograft tissue ${ }^{5}$. In-vivo screening for corneal lymphatics prior to transplantation could aid in the decision of when to perform surgery in high risk patients. Additionally, in-vivo detection of corneal lymph vessels posttransplantation could provide an early indicator of the degree of risk for rejection and subsequent transplant failure. Observation of cellular activity in lymph vessels may also contribute to a greater understanding of lymphatics in other areas of the body less amenable to non-invasive observation, such as cardiac, intestinal, and renal, and lung tissue ${ }^{22}$. Tumorassociated lymphangiogenesis and the metastasis of tumor cells via lymph vessels ${ }^{6,23}$ may also share cellular dynamics similar to those observed in corneal tissue. Additionally, studies examining the role of vascular endothelial growth factor-C in lymphangiogenesis as well as 
its therapeutic inhibition ${ }^{24}$ could benefit from the ability to study the same animals (and vessels) longitudinally.

Our technique of in-vivo lymphatic visualizaiton requires further refinement, specifically to determine the sensitivity and specificity of the technique in relation to ex-vivo methods. In the cases where lymphatics were observed prospectively, the path of the vessels was followed in an attempt to trace their entire course from the conjunctival lymphatics to the suture region. In practice, however, this was difficult due to the three-dimensional course of the vessels, and the presence of intervening blood vessels and non-transparent areas of ECM that served to obscure the lymphatics. Superficial lymphatics were the easiest to visualize. Further tests are required to determine the feasibility of in-vivo quantification of lymph vessel density. In conclusion, laser-scanning IVCM has been used to detect and monitor corneal lymph vessel activity in-vivo. The technique provides a means to study cellular activity within lymphatics and to conduct longitudinal observations of a given vessel, to complement ex-vivo visualization and analysis methods. Finally, we suggest that IVCM could be used to observe lymph vessels in human corneas, to be applied for example, in assessing the risk of corneal transplant failure.

\section{References}

1. Cursiefen C, Schlötzer-Schrehardt U, Küchle M, Sorokin L, Breiteneder-Geleff S, Alitalo $\mathrm{K}$, Jackson D. Lymphatic vessels in vascularized human corneas: immunohistochemical investigation using LYVE-1 and podoplanin. Invest Ophthalmol Vis Sci. 2002;43:21272135.

2. Cursiefen C, Maruyama K, Jackson DG, Streilein JW, Kruse FE. Time course of angiogenesis and lymphangiogenesis after brief corneal inflammation. Cornea 2006;25:443-447. 
3. Dana MR, Streilein JW. Loss and restoration of immune privilege in eyes with corneal neovascularization. Invest Ophthalmol Vis Sci. 1996;37:2485-2494.

4. Zhu S, Dana MR. Expression of cell adhesion molecules on limbal and neovascular endothelium in corneal inflammatory neovascularization. Invest Ophthalmol Vis Sci. 1999;40:1427-1434.

5. Yamagami S, Dana MR, Tsuru T. Draining lymph nodes play an essential role in alloimmunity generated in response to high-risk corneal transplantation. Cornea 2002;21:405-409.

6. Cao Y. Opinion: emerging mechanisms of tumor lymphangiogenesis and lymphatic metastasis. Nat Rev Cancer 2005;5:735-743.

7. Maruyama K, Ii M, Cursiefen C, et al. Inflammation-induced lymphangiogenesis in the cornea arises from CD11b-positive macrophages. J Clin Invest. 2005;115:2363-2372.

8. Ling S, Lin H, Liang L, Xu J, Xu C, Zhao W, Liu Z. Development of new lymphatic vessels in alkali-burned corneas. Acta Ophthalmol. 2009;87:315-322.

9. Ling S, Lin H, Xiang D, Feng G, Zhang X. Clinical and experimental research of corneal lymphangiogenesis after keratoplasty. Ophthalmologica 2008;222:308-316.

10. Guthoff RF, Zhivov A, Stachs O. In vivo confocal microscopy, an inner vision of the cornea - a major review. Clin Exp Ophthalmol. 2009;37:100-117.

11. Rasband, W.S., ImageJ. U. S. National Institutes of Health, Bethesda, Maryland, USA, http://rsb.info.nih.gov/ij/, 1997-2008.

12. Kirveskari J, Vesaluoma MH, Moilanen JAO, Tervo TMT, Petroll MW, Linnolahti E, Renkonen R. A novel non-invasive, in vivo technique for the quantification of leukocyte rolling and extravasation at sites of inflammation in human patients. Nat Med. $2001 ; 7: 376-379$ 
13. Labbé A, Liang H, Martin C, Brignole-Baudouin F, Warnet JM, Baudouin C.

Comparative anatomy of laboratory animal corneas with a new-generation high-resolution in vivo confocal microscope. Curr Eye Res. 2006;31:501-509.

14. Masters BR. Correlation of histology and linear and nonlinear microscopy of the living human cornea. J Biophoton. 2009;2:127-139.

15. Messmer EM, Mackert MJ, Zapp DM, Kampik A. In vivo confocal microscopy of pigmented conjunctival tumors. Graefe's Arch Clin Exp Ophthalmol. 2006;244:14371445.

16. De Nicola R, Labbé A, Amar N, Dupas B, Baudouin C. In vivo confocal microscopy and ocular surface diseases: anatomical-clinical correlations. J Fr Ophtalmol.2005;28:691698.

17. Guthoff RF, Baudouin C, Stave J. Atlas of Confocal Laser Scanning In-vivo Microscopy in Ophthalmology. Springer-Verlag, Berlin, 2006.

18. Berk DA, Swartz MA, Leu AJ, Jain RK. Transport in lymphatic capillaries. II. Microscopic velocity measurement with fluorescence photobleaching. Am J Physiol. 1996;270:H330-H337.

19. Mayrovitz HN, Larnard D, Duda G. Blood velocity measurement in human conjunctival vessels. Cardiovasc Dis. 1981;8:509-526.

20. Becker MD, Kruse FE, Joussen M, Rohrschneider, Nobiling R, Gebhard MM, Völcker HE. In vivo fluorescence microscopy of corneal neovascularization. Graefe's Arch Clin Exp Ophthalmol. 1998;236:390-398.

21. Messmer EM, Mackert MJ, Zapp DM, Kampik A. In vivo confocal microscopy of normal conjunctiva and conjunctivitis. Cornea. 2006 Aug;25(7):781-8) 
22. Schmid-Schonbein GW. Microlymphatics and lymph flow. Physiol Rev. 1990;70:9871028.

23. Skobe M, Hawighorst T, Jackson DG, et al. Induction of tumor lymphangiogenesis by VEGF-C promotes breast cancer metastasis. Nat Med. 2001;7:192-198.

24. Kubo H, Cao R, Bräkenhielm E, Mäkinen T, Cao Y, Alitalo K. Blockade of vascular endothelial growth factor receptor-3 signaling inhibits fibroblast growth factor-2-induced lymphangiogenesis in mouse cornea. Proc Natl Acad Sci USA. 2002;99:8868-8873. 
Table 1. Quantitative analysis of in-vivo morphologic parameters of corneal vessels.

\begin{tabular}{|c|c|c|c|c|c|c|c|}
\hline \multirow[b]{2}{*}{ Parameter } & \multicolumn{3}{|c|}{ Lymph vessels } & \multicolumn{3}{|c|}{ Blood vessels } & \multirow[b]{2}{*}{$\mathrm{P}$-value } \\
\hline & value & range & $\begin{array}{c}\text { no. of } \\
\text { meas }\end{array}$ & value & range & $\begin{array}{c}\text { no. of } \\
\text { meas }\end{array}$ & \\
\hline Vessel diameter $(\mu \mathrm{m})$ & $19.9 \pm 6.7$ & $12.5-43.8$ & 28 & $24.3 \pm 11.7$ & $6.3-62.5$ & 38 & 0.08 \\
\hline Lumen/ECM contrast ratio & $0.46 \pm 0.16$ & $0.18-0.74$ & 28 & $1.85 \pm 0.82$ & $0.68-4.81$ & 38 & $<0.001$ \\
\hline Leukocyte diameter $(\mu \mathrm{m})$ & $7.6 \pm 1.3$ & $4.7-10.3$ & 55 & $7.4 \pm 1.4$ & $5.2-11.6$ & 52 & 0.61 \\
\hline Cell velocity $(\mu \mathrm{m} / \mathrm{s})$ & $53 \pm 42$ & $3-140$ & 17 & $208 \pm 73$ & $125-425$ & 17 & $<0.001$ \\
\hline
\end{tabular}
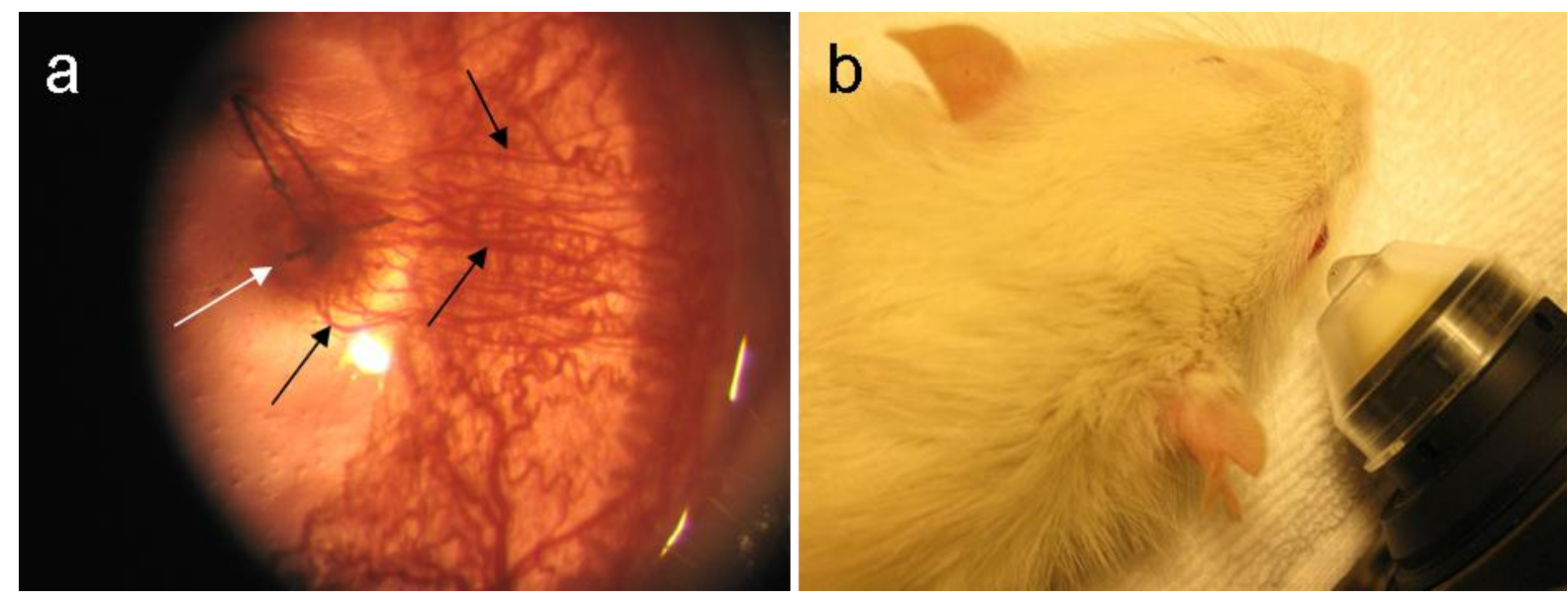

Figure 1. In-vivo examination of a corneal rat model of angiogenesis. a. neovascularization observed 14 days after suture placement (white arrow). Blood vessels (black arrows) originating from the limbus extend into the cornea toward the suture region (note: underlying vessels are in the iris and not in the cornea). b. a gel-coated confocal microscope objective lens is brought into contact with the rat cornea for in-vivo examination. 

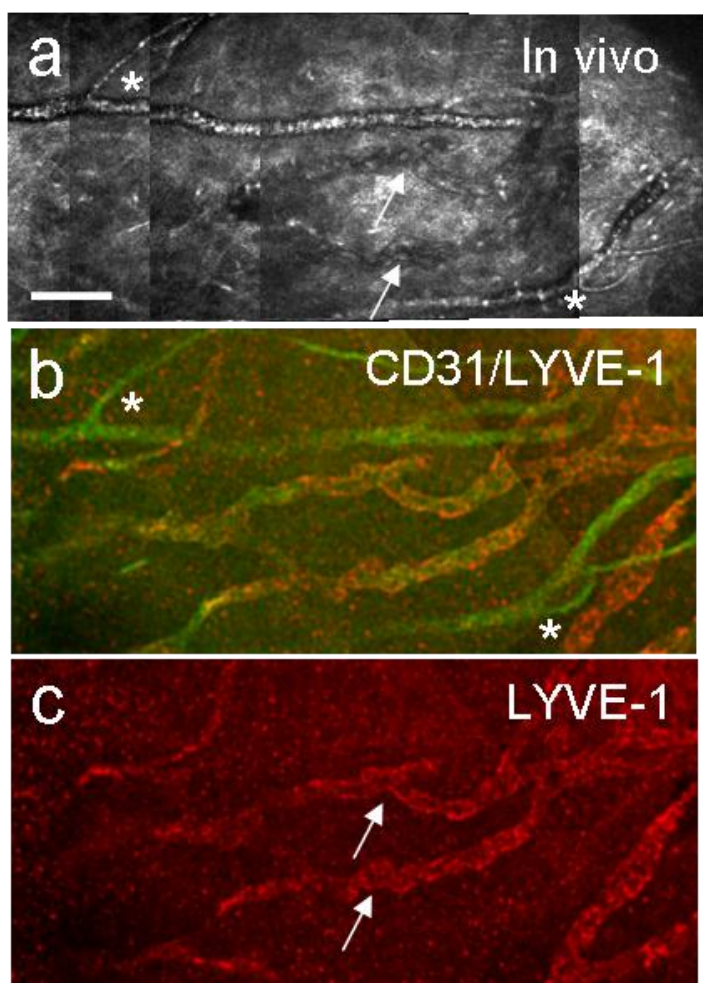

Figure 2. In-vivo and ex-vivo confocal image correlation. Retrospective comparison of the same corneal region with in-vivo confocal microscopy (a) and ex-vivo with immunofluorescent markers $(b, c)$. Features of blood vessels $(a, b, *)$ aided in the location of the same tissue region. LYVE-1 positive lymph vessels (c, arrows) were also visible in-vivo (a, arrows). Depth below corneal surface $=39 \mu \mathrm{m}$, bar $=100 \mu \mathrm{m}$.
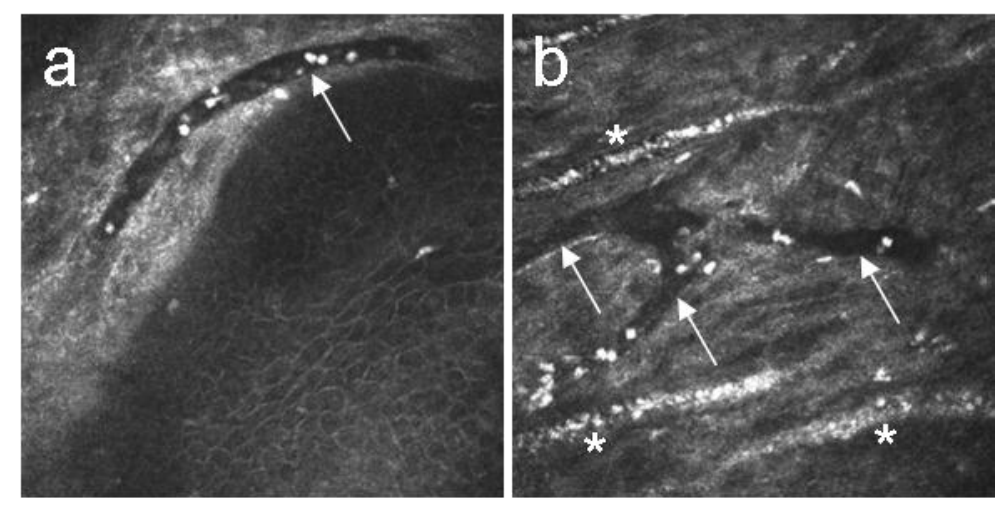

Figure 3. In-vivo confocal microscopy in a rat cornea. Presumed corneal lymphatics (a, b, arrows) observed in the rat cornea in-vivo 14 days after suture placement. Note the presence of reflective cells (leukocytes) within the vessels, and the presence of adjacent blood-filled vessels at the same corneal depth $\left(\mathrm{b},{ }^{*}\right)$. Depth below corneal surface $=43 \mu \mathrm{m}(\mathrm{a}), 42 \mu \mathrm{m}(\mathrm{b})$, images are $400 \times 400 \mu \mathrm{m}$. 


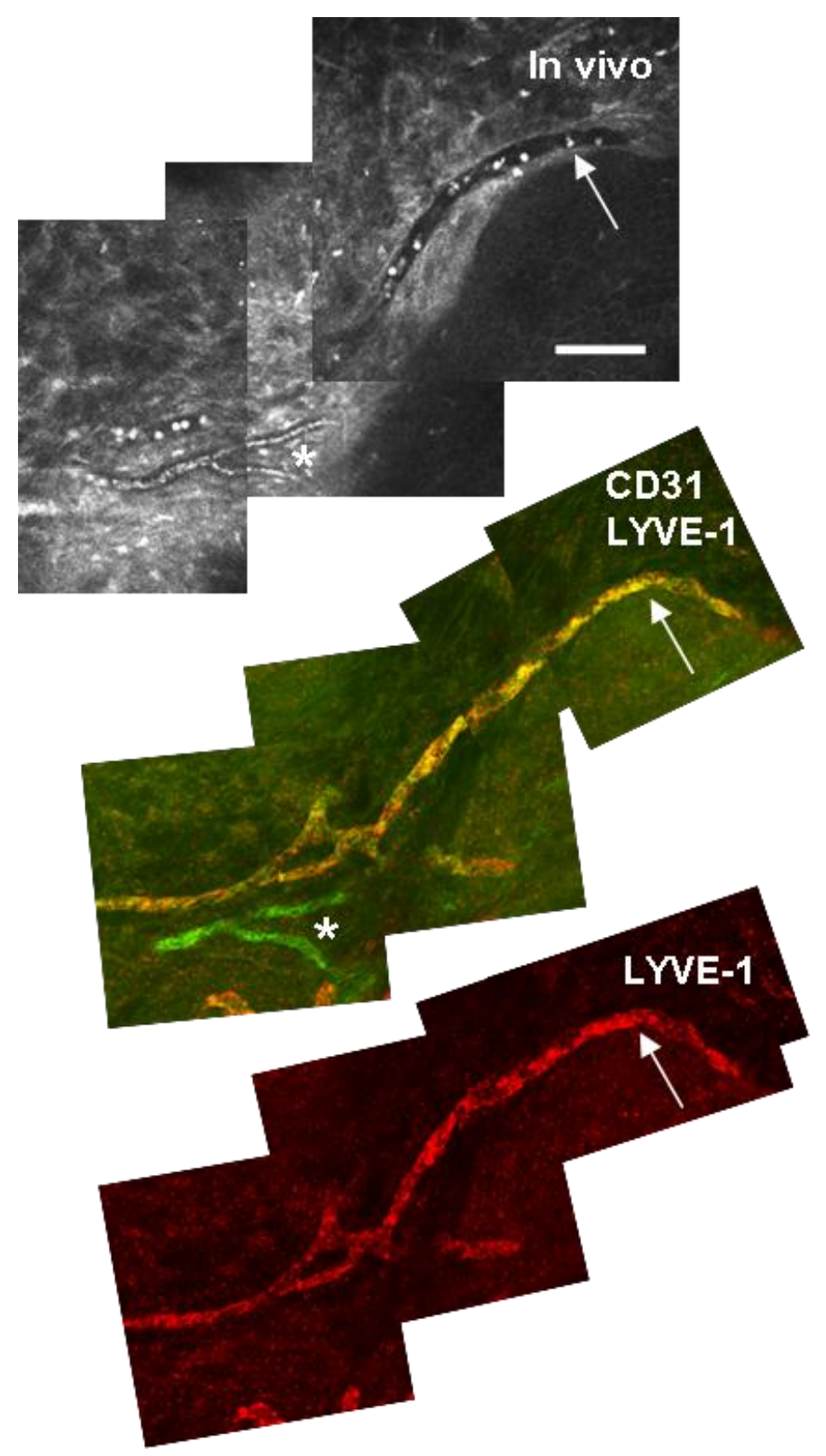

Figure 4. Immunofluorescence confirmation of presumed lymphatics in the rat cornea. The presumed lymphatic observed in-vivo (arrow) was confirmed as CD $31^{+} / \mathrm{LYVE}^{+{ }^{+++}}$, with adjacent CD31 $1^{+++} /$LYVE-1 $1^{-}$blood vessels $(*)$ serving as a point of reference. Depth below corneal surface $=43 \mu \mathrm{m}$, bar $=100 \mu \mathrm{m}$. 

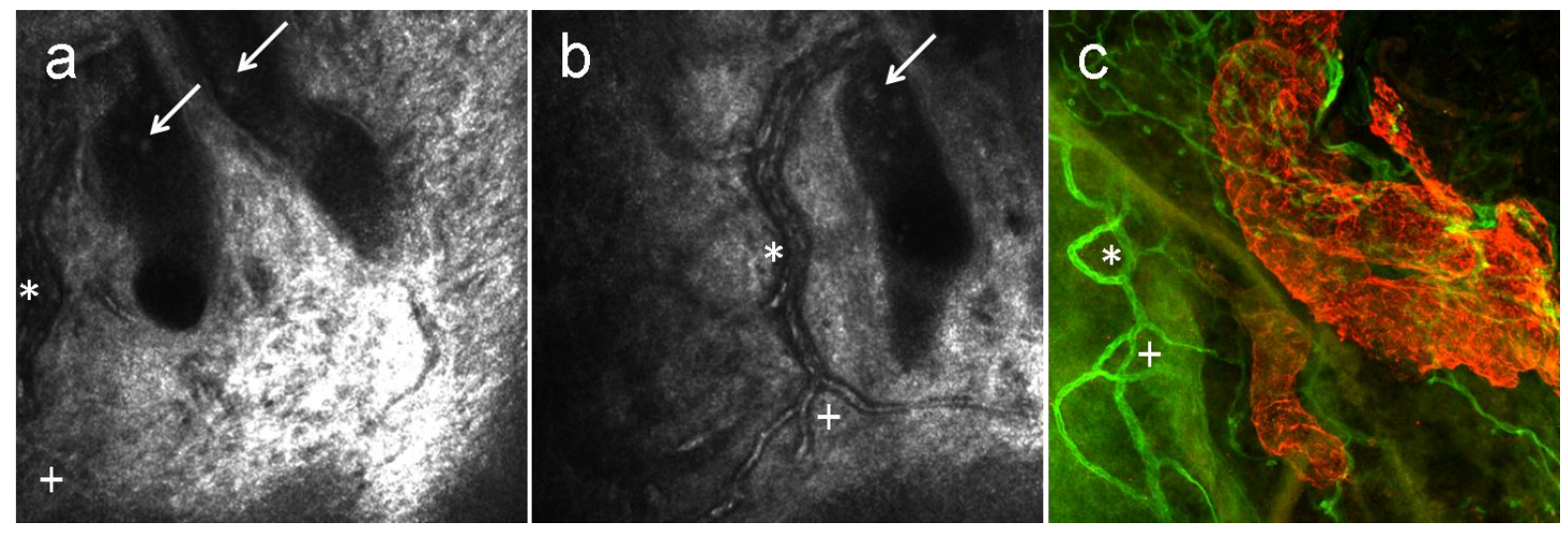

Figure 5. Immunofluorescence confirmation of lymphatics in the temporal perilimbal conjunctiva of a rat without corneal neovascularization. The same blood vessels seen in-vivo in the limbal region ( $a, b^{\text {'* }}$ ' and ' + ') were located after immunofluorescent staining with CD31/LYVE-1 (c, '*' and '+'). Conjunctival lymph vessels in-vivo had a wide, bulging dark lumen with only a few cells visible (a, b, arrows), compared to blood vessels exhibiting a smaller vessel diameter, thicker walls and a high number of small reflecting cells. Lymphatics were revealed by immunofluorescence to be part of a three-dimensional conjunctival lymphatic network (c, CD31 $1^{+} / \mathrm{LYVE}^{++++}$, red). Note the vessels in the limbal region ('*' and '+') appear with distortion in c due to flat-mount artefact. Depth below corneal surface $=$ $37 \mu \mathrm{m}(\mathrm{a}), 44 \mu \mathrm{m}(\mathrm{b})$, images are $400 \times 400 \mu \mathrm{m}$.

Supplementary Video 1 (Lymph Vessel 1.avi). Flow of blood in vessels and a leukocyte in a confirmed CD $31^{+} / \mathrm{LYVE} 1^{+++}$rat corneal lymph vessel (near top), viewed in-vivo without contrast enhancement.

Supplementary Video 2 (Lymph Vessel 2.avi). Slow movement of leukocytes within the confirmed rat corneal lymph vessel shown in Figure 4. 